\title{
Cochrane
}

Cochrane Database of Systematic Reviews

\section{Combination of magnetic resonance cholangiopancreatography and conventional magnetic resonance imaging for the diagnosis of bile duct stenosis (Protocol)}

Cavalcanti Neto AJ, Lustosa SAS, Casazza G, Reis C, Gomes MP, Do Carmo ACF, D'Ippolito G, Matos D

Cavalcanti Neto AJ, Lustosa SAS, Casazza G, Reis C, Gomes MP, Do Carmo ACF, D'Ippolito G, Matos D.

Combination of magnetic resonance cholangiopancreatography and conventional magnetic resonance imaging for the diagnosis of bile duct stenosis.

Cochrane Database of Systematic Reviews 2015, Issue 5. Art. No.: CD011692.

DOI: 10.1002/14651858.CD011692.

www.cochranelibrary.com

Combination of magnetic resonance cholangiopancreatography and conventional magnetic resonance imaging for the diagnosis of bile duct stenosis (Protocol) 
TABLE OF CONTENTS

HEADER . . . . . . . . . . . . . . . . . . . . . . . . . . . . . . . . . . . . . . . 1

ABSTRACT . . . . . . . . . . . . . . . . . . . . . . . . . . . . . . . . . . . . . . . 1

BACKGROUND . . . . . . . . . . . . . . . . . . . . . . . . . . . . . . . . . . . . . . . . . . . . . .

Figure 1. . . . . . . . . . . . . . . . . . . . . . . . . . . . . . . . . . . . . . 4

OBJECTIVES . . . . . . . . . . . . . . . . . . . . . . . . . . . . . . . . . . . . . . . . . . . . .

METHODS . . . . . . . . . . . . . . . . . . . . . . . . . . . . . . . . . . . . . . 5

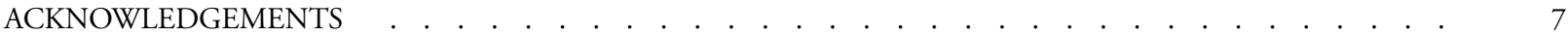

REFERENCES . . . . . . . . . . . . . . . . . . . . . . . . . . . . . . . . . . . . . 7

APPENDICES . . . . . . . . . . . . . . . . . . . . . . . . . . . . . . . . . . . . . .

WHAT'S NEW . . . . . . . . . . . . . . . . . . . . . . . . . . . . . . . . . . . . . 16

CONTRIBUTIONS OF AUTHORS . . . . . . . . . . . . . . . . . . . . . . . . . . . . . . . . . . . . . . . .

DECLARATIONS OF INTEREST . . . . . . . . . . . . . . . . . . . . . . . . . . . . . . . . . . . .

SOURCES OF SUPPORT . . . . . . . . . . . . . . . . . . . . . . . . . . . . . . . . . . . .

Combination of magnetic resonance cholangiopancreatography and conventional magnetic resonance imaging for the diagnosis of bile duct stenosis (Protocol)

Copyright $\odot 2015$ The Cochrane Collaboration. Published by John Wiley \& Sons, Ltd. 
[Diagnostic Test Accuracy Protocol]

\title{
Combination of magnetic resonance cholangiopancreatography and conventional magnetic resonance imaging for the diagnosis of bile duct stenosis
}

\author{
Augusto José Cavalcanti Neto ${ }^{1}$, Suzana AS Lustosa ${ }^{2}$, Giovanni Casazza ${ }^{3}$, Carolina Reis ${ }^{4}$, Marcelle Procopio Gomes ${ }^{5}$, Andreia Cristina \\ Feitosa Do Carmo ${ }^{6}$, Giuseppe D’Ippolito ${ }^{7}$, Delcio Matos ${ }^{8}$ \\ ${ }^{1}$ Surgical Department, UNIFESP-EPM, São Paulo, Brazil. ${ }^{2}$ Extension, Research, Teaching Unit - UEPE, Hospital Municipal Dr. \\ Munir Rafful, Volta Redonda, RJ, Brazil. ${ }^{3}$ Dipartimento di Scienze Biomediche e Cliniche “L. Sacco”, Università degli Studi di Milano, \\ Milan, Italy. ${ }^{4}$ School of Medical Sciences of Volta Redonda, UniFOA, Volta Redonda, Brazil. ${ }^{5}$ Escola de Ciências Médicas de Volta \\ Redonda, UniFOA, Volta Redonda, Brazil. ${ }^{6}$ Central Library, Escola Paulista de Medicina, Universidade Federal de São Paulo, São \\ Paulo, Brazil. ${ }^{7}$ Departamento de Diagnóstico por Imagem, Escola Paulista de Medicina, Universidade Federal de São Paulo, São Paulo, \\ Brazil. ${ }^{8}$ Department of Gastroenterological Surgery, Escola Paulista de Medicina, Universidade Federal de São Paulo, São Paulo, Brazil \\ Contact address: Augusto José Cavalcanti Neto, Surgical Department, Escola Paulista de Medicina, Universidade Federal de São Paulo, \\ Rua João Valiante 260 apto 102, Barra Mansa, Rio de Janeiro, 27323-210, Brazil. cavalcantiaj@gmail.com.
}

Editorial group: Cochrane Hepato-Biliary Group.

Publication status and date: Edited (no change to conclusions), published in Issue 5, 2015.

Citation: Cavalcanti Neto AJ, Lustosa SAS, Casazza G, Reis C, Gomes MP, Do Carmo ACF, D’Ippolito G, Matos D. Combination of magnetic resonance cholangiopancreatography and conventional magnetic resonance imaging for the diagnosis of bile duct stenosis. Cochrane Database of Systematic Reviews 2015, Issue 5. Art. No.: CD011692. DOI: 10.1002/14651858.CD011692.

Copyright (C) 2015 The Cochrane Collaboration. Published by John Wiley \& Sons, Ltd.

\begin{abstract}
A B S T R A C T
This is the protocol for a review and there is no abstract. The objectives are as follows:

To determine the diagnostic accuracy of the magnetic resonance imaging (MRI) and magnetic resonance cholangiopancreatography (MRCP) for detecting the presence of bile duct stenosis.

To evaluate the ability of the MRI and MRCP to determine the site of stenosis.

To evaluate the ability of MRI and MRCP to differentiate between benign and malignant bile duct stenosis.
\end{abstract}

\section{B A C K G R O U N D}

Bile duct stenosis is an abnormal stricture that causes impairment to the normal flow of bile on its way to the small bowel. Clinically, it manifests with jaundice, which represents with staining of the skin and sclerae by high blood levels of bilirubin (Friedman 2010). Among the causes for obstruction of the biliary system are stones, lymphadenopathy, benign tumours, primary biliary tu- mours, extension of other malignancies from other regions of the gastrointestinal tract, pancreatic masses, metastases to the liver, and cholangiocarcinoma. The treatment of bile duct stenosis depends on the type of obstruction, site, and extension of disease in question.

There are some difficulties in determining a rational strategy to evaluate people with jaundice by imaging methods. Jaundice is a

Combination of magnetic resonance cholangiopancreatography and conventional magnetic resonance imaging for the diagnosis of bile duct stenosis (Protocol)

Copyright $\odot 2015$ The Cochrane Collaboration. Published by John Wiley \& Sons, Ltd. 
clinical finding, not a single disease entity. The first task of the clinician caring for a person with jaundice is to determine if jaundice is caused by bile duct obstruction. People with a high pre-test probability of non-obstructive jaundice usually need no imaging investigations (ACR 1996).

It is an important challenge to differentiate between benign and malignant causes for biliary stenosis. Although the information acquired from cross-sectional imaging studies may suggest the most probable underlying cause, it is obscured in up to $50 \%$ of people (Yoo 2014). Indeed, the final diagnosis in people with stenosis without a demonstrable mass on an imaging investigation in crosssectional studies is difficult.

The imaging methods, currently used in evaluating obstructive jaundice, include abdominal ultrasonography, computed tomography, magnetic resonance imaging (MRI), magnetic resonance cholangiopancreatography (MRCP), endoscopic retrograde cholangiopancreatography (ERCP), and percutaneous transhepatic cholangiography (PTC) (Zhong 2005). These examinations are effective to varying degrees for assessing both the aetiology and site of obstructions.

Abdominal ultrasonography is the least invasive and cheapest imaging technique available for evaluating obstructive jaundice. It is a quick and easy test, suitable for initial diagnostic imaging. Its sensitivity ranges between $55 \%$ and $95 \%$ and specificity between $71 \%$ and $96 \%$ (Yusoff 2003). Ultrasound is less effective than computed tomography and MRCP for determining the site and cause of biliary obstruction (Yusoff 2003).

Computed tomography is slightly more sensitive (74\% to $96 \%$ ) and specific ( $90 \%$ to $94 \%)$ than ultrasound for detecting biliary stenosis (ACR 1996).

MRI can demonstrate both the site and cause of stenosis. MRCP is useful in depicting the biliary anatomy (ACR 1996). The combination of MRI and MRCP represents another non-invasive imaging modality showing an excellent soft tissue contrast and having the ability to demonstrate pathological and congenital conditions (Yoo 2014).

ERCP is the most common invasive diagnostic biliary procedure. Due to significant advances in cross-sectional imaging, in particular the advent of MRCP, the current role of ERCP is exclusively therapeutic (ACR 1996).

One study evaluating tumour extension and potential resectability of bile duct cancer concluded that the findings of MRCP combined with the MRI findings are comparable with the multi detector computed tomography (MDCT) and direct cholangiography (Park 2008).

People with suspected stenosis require an effective diagnostic test that can differentiate between low-risk malignant disease that could be cured with minimally invasive therapies and high-risk malignant disease that would benefit from surgical exploration. Thus, the diagnosis of the cause of biliary stricture has an important role in optimising the treatment. It seems that combination of MRI and MRCP could be used for differentiating causes of benign and malignant biliary strictures.

\section{Target condition being diagnosed}

\section{Bile duct stenosis - presence, site, and aetiology (benign versus malignant stenosis)}

Biliary strictures, biliary stenosis, or bile duct stenosis are synonymous. Stenosis is defined as a constriction or narrowing of a duct or passage (The American Heritage Dictionary). Many diseases could lead to a biliary stenosis.

Diseases that lead to stenosis could affect the biliary ducts in any site of the biliary tree, from the small ducts in the periphery of the liver to the distal portion of choledochal duct.

Choledocholithiasis and malignant bile duct tumours are the most common diseases that involve the biliary system, and pancreatitis and pancreatic carcinoma are the most common disorders of the pancreas (Reinhold 1996). All of these diseases could lead to direct or indirect stenosis of the biliary tree.

Benign and malignant stenosis have different imaging presentations. Abrupt termination of the bile duct is a cholangiographic sign that has a high correlation with malignancy, whereas a gradually tapering duct correlates with a benign process. These features can be visualised through cholangiographic images or with correlation with sequential axial images. The key to achieving a proper diagnosis of benign or malignant stenosis is to analyse the transition zone between the stenotic and non-stenotic area. Some radiologists recognise the need to evaluate axial T1-weighted and T2-weighted images sequentially for complete evaluation of the transition zone of the bile duct obstruction for extra-ductal and intraductal findings (Baron 2002).

\section{Index test(s)}

\section{Magnetic resonance cholangiopancreatography and magnetic resonance imaging}

The index test will be a combination of sequences of MRI using both MRCP and MRI of the abdomen.

$\mathrm{MRI}$ is a non-invasive imaging technique, which uses a magnetic field that can produce imaging of the whole body. These images have a good contrast between the different tissues. MRI permits combining the benefits of cross-sectional and projection techniques, without moving the person. MRI does not use radiation. MRCP is a non-invasive test that can produce images of the biliary tree. This technique is based on heavily T2-weighted pulse 
sequences. As a result, stationary fluids have a high signal intensity, while solid organs have a low signal intensity (Reinhold 1996).

MRCP images can be obtained using a variety of pulse sequences. HASTE (half-Fourier acquisition single-shot turbo spin-echo), Gradient-Echo (Steady-State Free Precession Signals - SSFP), and RARE (rapid acquisition with relaxation enhancement sequences) are pulse sequences available for studies of biliary and pancreatic ducts (Miyazaki 1996; Reinhold 1996; Patel 2009). Breath-hold HASTE MRCP can be used as a non-invasive screening method for pancreaticobiliary diseases (Miyazaki 1996). MRCP can provide diagnostic information equivalent to invasive techniques in a large percentage of people (Barish 1995).

We will consider the index test positive for biliary stenosis if any narrowing in the biliary tree, associated or not to biliary dilation, is shown on the image. We will consider the index test negative for stenosis in the cases when the diameter of the bile duct is not reduced.

\section{Clinical pathway}

People with jaundice, leading to cholestasis, usually undergo diagnostic laboratory tests such as bilirubin, alkaline phosphatases, or gamma-glutamyl transpeptidase to differentiate obstructive from non-obstructive jaundice. People with high probability of nonobstructive jaundice usually do not undergo imaging tests. People with tests suggesting obstructive jaundice, usually undergo further investigation.

With the availability of newer imaging and sampling methods, algorithms for diagnostic evaluation and management of people with suspected biliary obstruction evolved. ERCP is no longer routinely recommended and hence, it is not the initial test of choice. Biliary strictures remain a diagnostic enigma and the possibility of achieving an early and accurate diagnosis is high. While abdominal imaging helps in finding the level of obstruction and provides a 'road map' for further endoscopic investigations, tissue diagnosis is usually needed to make decisions on management (Singh 2015) (Figure 1). 
Figure I. Clinical pathway:Proposed diagnostic approach to biliary strictures (modified from Singh

20 I5).EUS-FNA: endoscopic ultrasound-fine needle aspiration; ERCP: endoscopic retrograde cholangiopancreatography; IDUS: intraductal ultrasound; MRI/MRCP: magnetic resonance imaging/magnetic resonance cholangiopancreatography; SOC: single operator cholangioscopy; US: ultrasound. For results with low likelihood of malignancy, close observation and follow-up are indicated.* Once malignancy is confirmed by any modality, surgical and oncology referral should be obtained.

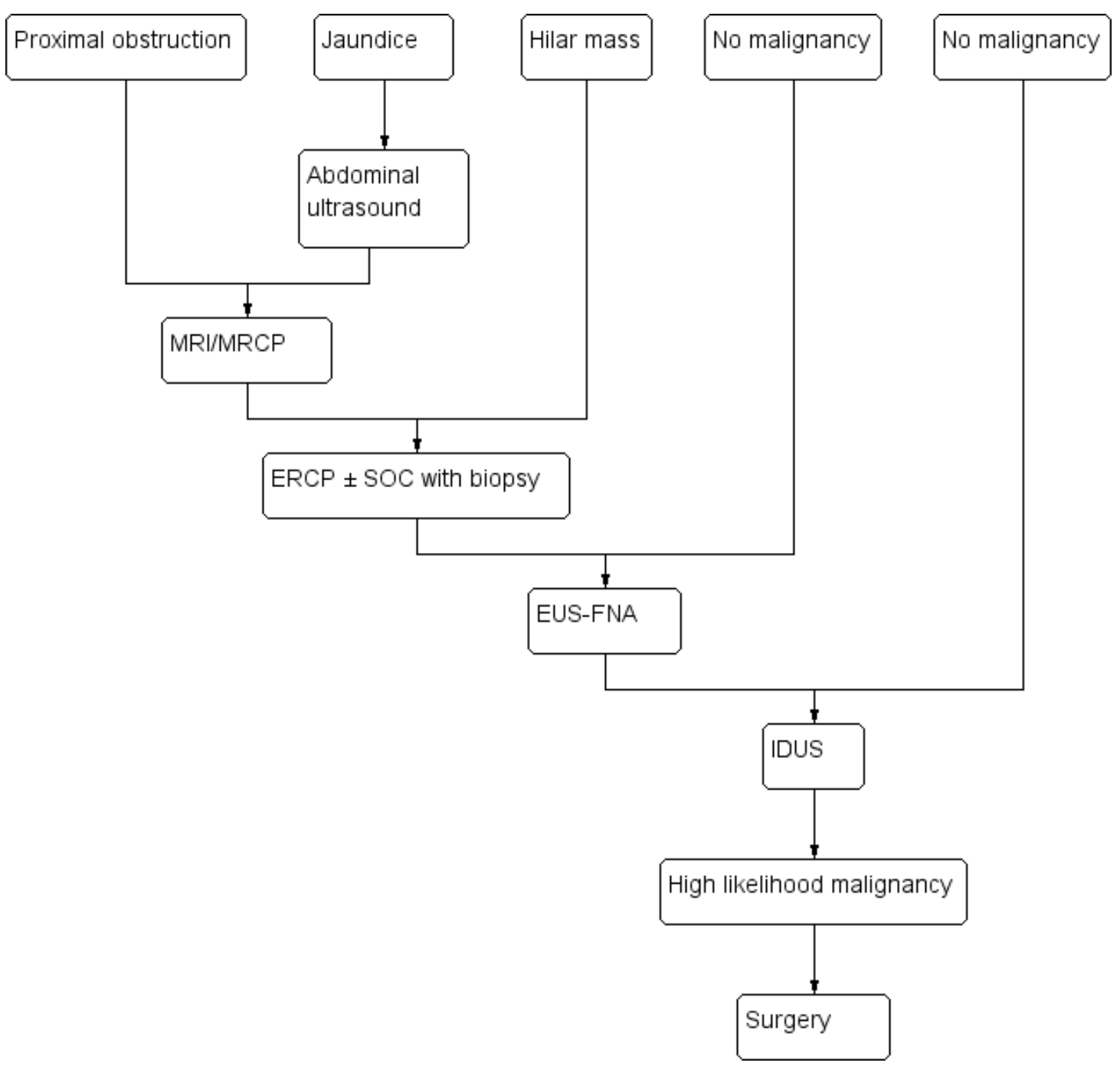

Combination of magnetic resonance cholangiopancreatography and conventional magnetic resonance imaging for the diagnosis of bile duct stenosis (Protocol)

Copyright $\Subset 2015$ The Cochrane Collaboration. Published by John Wiley \& Sons, Ltd. 


\section{Alternative test(s)}

The imaging methods, currently used in evaluating obstructive jaundice, include abdominal ultrasonography, computed tomography, MRI, MRCP, ERCP, and PTC. These imaging methods are effective to varying degrees for assessing presence, site, and aetiology of stenosis. The sequence in which these tests are used is not standardised (Mosler 2011).

In people with mild symptoms, less invasive tests (e.g., transabdominal ultrasound, computed tomography, MRI, MRCP, or endoscopic ultrasound) are often used to establish diagnosis and guide further management. The recent literature has emphasised that more invasive modalities should be reserved for people with an indication for therapeutic intervention (Mosler 2011).

\section{Rationale}

We consider that the combination of the magnetic resonance techniques - MRCP and MRI - may improve the pathway of diagnosis of diseases that cause stenosis of the biliary tree (Haliloglu 2009). The combination of MRCP and MRI allows an accurate imaging of the site, extension, and cause of stenosis (Catalano 1998; Hänninen 2002; Hänninen 2005). These magnetic resonance techniques could be used to direct properly people to ERCP and to plan a certain therapy. Some study authors have already demonstrated that the use of MRCP plus MRI significantly improves the diagnostic accuracy of MRI examinations for diseases of the biliary system (Kim 2000; Kim 2006; Kim 2007).

In the cases when an ultrasound investigation result is negative, based on a high pre-test probability of biliary obstruction, further tests are necessary for the management of people with cholestasis (ACR 1996). Many factors could be responsible for a false-negative ultrasound result; for example, the absence of biliary dilation in the presence of obstruction or stenosis, the expertise of the examiner, or the inability to visualise the extrahepatic biliary tree (Baron 2002).

Furthermore, as previously stated, there is no standardisation of the imaging method for the diagnostic evaluation of people with biliary stenosis (Mosler 2011).

\section{O B JECT IVES}

To determine the diagnostic accuracy of the magnetic resonance imaging (MRI) and magnetic resonance cholangiopancreatography (MRCP) for detecting the presence of bile duct stenosis.

\section{Secondary objectives}

To evaluate the ability of the MRI and MRCP to determine the site of stenosis.

To evaluate the ability of MRI and MRCP to differentiate between benign and malignant bile duct stenosis.

\section{METHODS}

\section{Criteria for considering studies for this review}

\section{Types of studies}

We will include prospective and retrospective studies assessing the diagnostic accuracy of MRCP plus MRI in consecutive series of people with suspected bile duct stenosis. We will include studies irrespective of publication status or language. We will not include participant/control design studies. We will exclude studies using MRCP alone as we intend to verify the accuracy of the combination of MRCP plus MRI.

\section{Participants}

Adults of either sex with suspicion of biliary stenosis, symptomatic or asymptomatic, with laboratory changes, that is, changes in serum levels of bilirubin, alkaline phosphatase, and gamma glutamyl transferase above the normal range. People with symptoms could present with cholestasis, defined by jaundice, choluria, and acholic stools. They could also present with upper right abdominal pain, fever, and weight loss. We will also consider inclusion of data from people with or without symptoms, if they were investigated using abdominal ultrasonography before the index test.

\section{Index tests}

The index test will be the combination of MRCP plus MRI techniques.

\section{Target conditions}

Target conditions will be: presence, site, and nature (benign or malignant) of stenosis. We plan to include studies on any of these three target conditions as well as studies on the presence, location, or cause of stenosis.

We will define bile duct stenosis as any degree of narrowing of the diameter of the biliary ducts observed by the use of the reference standard. 
We will dichotomise the site of the biliary stenosis in two categories: intrahepatic and extrahepatic. We will define intrahepatic stenosis as narrowing in the biliary tree inside the liver, including the common hepatic duct. We will define extrahepatic stenosis as narrowing at any portion of the choledochal duct.

The nature of bile duct stenosis will be categorised as benign and malignant. A malignant bile duct stenosis is produced by a primary biliary cancer or extension of other malignancies from different regions of the body (e.g., cholangiocarcinoma, liver metastasis, pancreatic adenocarcinoma) and a benign biliary stenosis is produced by causes different from malignant neoplasm, such as inflammatory process, papillary changes, and benign neoplasms. To differentiate the nature of stenosis with the index test, we will analyse the transition zone between the stenotic and non-stenotic area. A malignant stenosis is represented by an abrupt termination between the stenotic and non-stenotic duct. Benign stenosis is represented by a gradually tapering duct.

\section{Reference standards}

The reference standard is ERCP alone or in a combination with any of the following diagnostic tests: intraoperative cholangiopancreatography, PTC, endoscopic ultrasonography, surgical exploration, or histopathology. We will also consider accepting as a reference standard a follow-up of participants with a negative index test for a period of no less than one month.

ERCP alone, or ERCP in combination with PTC and endoscopic ultrasonography tests will be the reference standard(s) to define the presence and site of stenosis.

The combination of clinical follow-up, surgical exploration, and histopathology will be the reference standard to differentiate between benign and malignant causes of stenosis.

\section{Search methods for identification of studies}

\section{Electronic searches}

We will search The Cochrane Hepato-Biliary Controlled Trials Register and The Cochrane Hepato-Biliary Diagnostic Test of Accuracy Studies Register, The Cochrane Library, MEDLINE, EMBASE, Science Citation Index, and LILACS (Royle 2003). We will apply no language or document type restrictions.

We list the preliminary search strategies with the expected time spans of the searches in Appendix 1.

\section{Searching other resources}

We will also screen reference list of the included studies.

\section{Data collection and analysis}

Two review authors (AJCN and SASL) will review the abstracts of the retrieved publications and select those that are likely to meet the inclusion criteria. Four review authors (AJCN, SASL, CR, and MPG) will independently collect data for analyses. If there are disagreements, the authors will discuss these until they reach consensus.

\section{Selection of studies}

A study publication has to be an assessment of the accuracy of the index test in people with suspected biliary stenosis. Four review authors (AJCN, SASL, CR, and MPG) will independently review the publications for further data extraction.

\section{Data extraction and management}

Two review authors (AJCN, SASL) will independently extract data from all included studies using a data collection form. Three review authors (AJCN, SASL, and GD) will deal critically with the results and will resolve any disagreements by checking the publication study data.

Data extraction will include study design, year of publication, size of sample, data for $2 \times 2$ tables (true positives, false positives, true negatives, false negatives) or data for $3 \times 2$ tables, withdrawals and reasons, reference standard, diseases, strength of magnetic field, and assessment of all the items of Quality Assessment of Diagnostic Accuracy Studies (QUADAS-2) in terms of bias risk of the studies (Whiting 2011).

We also intend to extract data for people with or without abdominal ultrasonography investigation before the index test. Ultrasound imaging results could have an effect on diagnostic pre-test probability. Participants with ultrasound-positive results could affect the accuracy of the index test and the reference standard. We will consider these results as a source of heterogeneity.

We will extract data for $2 \times 2$ tables to evaluate the ability of the index test to differentiate the site of bile duct stenosis as intrahepatic or extrahepatic.

In the case of indeterminate index test results and if the primary studies allow, we will extract data for a $3 \times 2$ table (true positives, false positives, true negatives, false negatives, non-evaluable results). The 'classic' $2 \times 2$ table does not hold enough information to show the true range of possible results. By simply excluding nonevaluable results, the values of sensitivity and specificity become overestimated (Schuetz 2012).

\section{Assessment of methodological quality}

We will assess the selected studies using QUADAS-2, as recommended by the Cochrane Handbook for Systematic Reviews of Diagnostic Test Accuracy (still in draft) (DTA Handbook 2009). 
Three review authors (AJCN, SASL, and GD) will independently assess the quality of the studies, and resolve any disagreements by consensus.

Appendix 2 presents a detailed definition of criteria for signalling questions, risk of bias, and concerns about applicability of the four domains of QUADAS-2.

We will consider studies at low risk of bias only if the studies are classified as low risk of bias considering all the QUADAS-2 domains.

\section{Statistical analysis and data synthesis}

We will create a $2 \times 2$ table of the diagnostic accuracy of MRI plus MRCP for diagnosing the presence, or the site, or the aetiology (benign versus malignant) of biliary stenosis. We will report sensitivity, specificity, and positive and negative likelihood ratios (LR+ and LR-) with their 95\% confidence intervals (CI) for each primary study. We will perform a graphical descriptive analysis of the included studies with forest plots (of sensitivity and specificity) and with graphical representation of the studies in the receiver operating characteristic (ROC) space (plot of sensitivity versus 1 specificity). We will fit the bivariate model fitted (Reitsma 2005), and use the results to calculate the pooled estimates of sensitivity and specificity (mean operating point). We will obtain summary estimates of LRs from model-derived sensitivity and specificity estimates.

If primary studies present non-evaluable results about the index test, we will create a $3 \times 2$ table of diagnostic accuracy of index test for bile duct stenosis. We will then adopt the intention-to-diagnose approach to build $2 \times 2$ tables for meta-analyses. According to this approach, we will include non-evaluable results either in the false-negative or false-positive cell of the $2 \times 2$ table, as appropriate (Schuetz 2012).

If possible, we will add some relevant co-variates to the bivariate model to investigate the effect of the pre-defined sources of heterogeneity on the accuracy estimates (Investigations of heterogeneity). We will perform statistical analyses with statistical software SAS, release 9.2.

\section{Investigations of heterogeneity}

We will investigate heterogeneity in a descriptive way (forest plots for various subgroups of studies), as well as in a more formal way, by adding co-variates to the bivariate model.

We consider the following as potential sources of heterogeneity.

- MRCP technological differences in the magnetic strength field (0.5 Tesla (T) versus 1.0 T versus 1.5 T).

- Type of reference standard. We will define three categories:

o direct cholangiographies: represented by ERCP,

intraoperative cholangiopancreatography, PTC;

○ surgical types: represented by surgical exploration,

histopathological findings, and clinical; and

$\circ$ other imaging tests: represented by clinical follow-up

and endoscopic ultrasonography.

- Clinical context (studies with people not undergoing surgery versus studies with people undergoing surgery).

- Participants submitted to other imaging tests before the index test or reference standard, including people with positive transabdominal ultrasound.

- Type of disease that led to stenosis.

\section{Sensitivity analyses}

We will undertake sensitivity analyses to explore the effect on overall results of studies with high risk of bias, by including only the studies classified at low risk of bias in the analyses.

\section{ACKNOW LEDGEMENTS}

Dimitrinka Nikolova and Agostino Colli for help and answers on all queries during the preparation of the review protocol.

Peer reviewers: anonymous - names known by The Cochrane Diagnostic Test Accuracy Reviews Editorial Team, UK.

Contact editor from The Cochrane Hepato-Biliary Group: Agostino Colli, Italy.

\section{R E F E R E N C E S}

\section{Additional references}

\section{ACR 1996}

American College of Radiology. ACR Appropriateness Criteria: jaundice. www.acr.org/ /media/ACR/ Documents/AppCriteria/Diagnostic/Jaundice.pdf 1996 (accessed 31 March 2015).

Barish 1995

Barish MA, Yucel EK, Soto JA, Chuttani R, Ferrucci JT. MR cholangiopancreatography: efficacy of three- dimensional turbo spin-echo technique. AJR. American Journal of Roentgenology 1995;165:295-300.

\section{Baron 2002}

Baron RL, Tublin ME, Peterson MS. Imaging the spectrum of biliary tract disease. Radiologic Clinics of North America 2002;40:1325-54.

\section{Catalano 1998}

Catalano C, Pavone P, Laghi A, Panebianco V, Scipioni

A, Fanelli F, et al. Pancreatic adenocarcinoma: combination of MR imaging, MR angiography and MR 
cholangiopancreatography for the diagnosis and assessment of resectability. European Radiology 1998;8:428-34.

\section{DTA Handbook 2009}

Reitsma JB, Rutjes AWS, Whiting P, Vlassov VV, Leeflang MMG, Deeks JJ. Chapter 9: Assessing methodological quality. In: Deeks JJ, Bossuyt PM, Gatsonis C (editors), Cochrane Handbook for Systematic Reviews of Diagnostic Test Accuracy Version 1.0.0. The Cochrane Collaboration, 2009. Available from: srdta.cochrane.org/ (accessed 31 March 2015).

Friedman 2010

Friedman LS. Liver, biliary tract, \& pancreas disorders. In: Mcphee SJ, Papadakis MA editor(s). Current Medical Diagnosis \& Treatment. 9th Edition. Maidenhead, UK: The McGraw-Hill Companies Inc., 2010:598-648.

Haliloglu 2009

Haliloglu N, Erden A, Erden I. Primary biliary cirrhosis: evaluation with T2-weighted MR imaging and MR cholangiopancreatography. European Journal of Radiology 2009;69(3):523-7.

\section{Hänninen 2002}

Lopez Hänninen E, Amthauer H, Hosten N, Ricke J, Böhmig M, Langrehr J, et al. Prospective evaluation of pancreatic tumors: accuracy of MR imaging with MR cholangiopancreatography and MR angiography. Radiology 2002;224:34-41.

\section{Hänninen 2005}

Hänninen EL, Ricke J, Amthauer H, Röttgen R, Böhmig M, Langrehr J, et al. Magnetic resonance cholangiopancreatography: image quality, ductal morphology, and value of additional T2- and T1-weighted sequences for the assessment of suspected pancreatic cancer. Acta Radiologica 2005;46:117-25.

Kim 2000

Kim MJ, Mitchell DG, Ito K, Outwater EK. Biliary dilatation: differentiation of benign from malignant causes - value of adding conventional MR imaging to MR cholangiopancreatography. Radiology 2000;214(1):173-81.

Kim 2006

Kim YK, Kim CS, Lee JM, Ko SW, Chung GH, Lee $\mathrm{SO}$, et al. Value of adding T1-weighted image to MR cholangiopancreatography for detecting intrahepatic biliary stones. AJR. American Journal of Roentgenology 2006;187: W267-74.

\section{Kim 2007}

Kim JY, Lee JM, Han JK, Kim SH, Lee JY, Choi JY, et al. Contrast-enhanced MRI combined with MR cholangiopancreatography for the evaluation of patients with biliary strictures: differentiation of malignant from benign bile duct strictures. Journal of Magnetic Resonance Imaging 2007;26:304-12.

\section{Miyazaki 1996}

Miyazaki T, Yamashita Y, Tsuchigame T, Yamamoto H, Urata J, Takahashi M. MR cholangiopancreatography using HASTE (Half-Fourier Acquisition Single-Shot Turbo Spin-
Echo) sequences. AJR. American Journal of Roentgenology 1996;166:1297-303.

Mosler 2011

Mosler P. Diagnosis and management of acute cholangitis. Current Gastroenterology Reports 2011;13:166-72.

\section{Park 2008}

Park HS, Lee JM, Choi JY, Lee MW, Kim HJ, Han JK, Choi BI. Preoperative evaluation of bile duct cancer: MRI combined with MR Cholangiopancreatography versus MDCT with direct cholangiography. AJR 2008;190: 396-405.

\section{Patel 2009}

Patel HT, Shah AJ, Khandelwal SR, Patel HF, Patel MD. MR Cholangiopancreatography at 3.0 T. Radiographics 2009;29(6):1689-706.

\section{Reinhold 1996}

Reinhold C, Bret PM. Current status of MR cholangiopancreatography. AJR. American Journal of Roentgenology 1996;166:1285-95.

\section{Reitsma 2005}

Reitsma JB, Glas AS, Rutjes AW, Scholten RJ, Bossuyt $\mathrm{PM}$, Zwinderman $\mathrm{AH}$. Bivariate analysis of sensitivity and specificity produces informative summary measures in diagnostic reviews. Journal of Clinical Epidemiology 2005; 58(10):982-90.

\section{Royle 2003}

Royle P, Milne R. Literature searching for randomized controlled trials used in Cochrane reviews: rapid versus exhaustive searches. International Journal of Technology Assessment in Health Care 2003;19(4):591-603.

\section{Schuetz 2012}

Schuetz GM, Schlattmann P, Dewey M. Use of 3x2 tables with an intention to diagnose approach to assess clinical performance of diagnostic tests: meta-analytical evaluation of coronary CT angiography studies. BMJ (Clinical Research Ed.) 2012;345:e6717.

\section{Singh 2015}

Singh A, Gellrud A, Agarwal B. Biliary strictures: diagnostic considerations and approach. Gastroenterology Report 2015; 3(1):22-31.

The American Heritage Dictionary

The American Heritage Dictionary. Stenosis. www.ahdictionary.com/word/search.html?q=stenosis (accessed 31 March 2015).

Whiting 2011

Whiting PF, Rutjes AW, Westwood ME, Mallett S, Deeks JJ, Reitsma JB, et al. QUADAS-2: a revised tool for the quality assessment of diagnostic accuracy studies. Annals of Internal Medicine 2011;155:529-36.

Yoo 2014

Yoo RE, Lee JM, Yoon JH, Kim JH, Han JK, Choi BI. Differential diagnosis of benign and malignant distal biliary strictures: value of adding diffusionweighted imaging to conventional magnetic resonance 
cholangiopancreatography. Journal of Magnetic Resonance

Imaging 2014;39(6):1509-17.

\section{Yusoff 2003}

Yusoff IF, Barkun JS, Barkun AN. Diagnosis

and management of cholecystitis and cholangitis.

Gastroenterology Clinics of North America 2003;32:1145-68.

\section{Zhong 2005}

Zhong L, Li L, Yao QY. Preoperative evaluation of

pancreaticobiliary tumor using MR multi-image techniques.

World Journal of Gastroenterology 2005;11(24):3756-61.

* Indicates the major publication for the study

\section{A P P E N D I C ES}

\section{Appendix I. Search strategies}

\begin{tabular}{|c|c|c|}
\hline Database & Time span & Search strategy \\
\hline $\begin{array}{l}\text { Cochrane Hepato-Biliary Controlled Trials } \\
\text { Register }\end{array}$ & Date will be given at review stage. & $\begin{array}{l}\text { ((((Bile AND (duct OR tract)) OR biliary OR } \\
\text { pancreatic* OR biliopancreatic*) AND (tumor* } \\
\text { OR lesion* OR injur* OR strictur* OR disease* } \\
\text { OR disorder* OR obstruction* OR stenos*)) OR } \\
\text { (Chronic pancreatitis OR cholangitis OR focal } \\
\text { wall thickening OR choledochal varices OR (por- } \\
\text { tal AND (biliopathy OR cavernoma)) OR obstruc- } \\
\text { tive jaundice OR bile leakage OR retained stones } \\
\text { OR (bile duct AND (intrahepatic OR aberrant)) } \\
\text { OR intraductal masses OR duct lumen)) AND ( } \\
\text { ((NMR OR MR OR protonspin OR magnetiza- } \\
\text { tion OR MRI OR chemical shift) AND (imag- } \\
\text { ing OR tomography OR scan)) OR (magnetic res- } \\
\text { onance cholangiopancreatograph* OR zeugmato- } \\
\text { graph* OR functional MRI)) }\end{array}$ \\
\hline
\end{tabular}

Cochrane Hepato-Biliary Diagnostic Test Date will be given at review stage. of Accuracy Studies Register

((((Bile AND (duct OR tract)) OR biliary OR pancreatic* OR biliopancreatic*) AND (tumor* OR lesion* OR injur* OR strictur* OR disease* OR disorder* OR obstruction* OR stenos*)) OR (Chronic pancreatitis OR cholangitis OR focal wall thickening OR choledochal varices OR (portal AND (biliopathy OR cavernoma)) OR obstructive jaundice OR bile leakage OR retained stones OR (bile duct AND (intrahepatic OR aberrant)) OR intraductal masses OR duct lumen)) AND ( 
((NMR OR MR OR protonspin OR magnetization OR MRI OR chemical shift) AND (imaging OR tomography OR scan)) OR (magnetic resonance cholangiopancreatograph* OR zeugmatograph* OR functional MRI))

\#1 MeSH descriptor: [Bile Ducts] explode all trees \#2 MeSH descriptor: [Biliary Tract Diseases] explode all trees

\#3 MeSH descriptor: [Adenoma, Bile Duct] explode all trees

\#4 MeSH descriptor: [Choledochal Cyst] explode all trees

\#5 MeSH descriptor: [Pancreatic Neoplasms] explode all trees

\#6 MeSH descriptor: [Pancreatitis] explode all trees \#7 MeSH descriptor: [Cholangiocarcinoma] explode all trees

\#8 MeSH descriptor: [Carcinoma, Hepatocellular] explode all trees

\#9 MeSH descriptor: [Jaundice, Obstructive] explode all trees

\#10 MeSH descriptor: [Liver Cirrhosis] explode all trees

\#11 ((((Bile and (duct or tract)) or biliary or pancreatic* or biliopancreatic*) and (tumor* or lesion* or injur* or strictur* or disease* or disorder* or obstruction* or stenos*)) or (Chronic pancreatitis or cholangitis or focal wall thickening or choledochal varices or (portal and (biliopathy or cavernoma)) or obstructive jaundice or bile leakage or retained stones or (bile duct and (intrahepatic or aberrant)) or intraductal masses or duct lumen))

$\# 12 \# 1$ or $\# 2$ or $\# 3$ or $\# 4$ or $\# 5$ or $\# 6$ or $\# 7$ or $\# 8$ or \#9 or \#10 or \#11

\#13 MeSH descriptor: [Magnetic Resonance Imaging] explode all trees

\#14 (((NMR or MR or protonspin or magnetization or MRI or chemical shift) and (imaging or tomography or scan)) or (magnetic resonance cholangiopancreatograph* or zeugmatograph* or functional MRI))

\#15 \#13 OR \#14

\#16 \#12 AND \#15

\# 1 'nuclear magnetic resonance imaging': ab,ti \# 2 'nuclear magnetic resonance imaging'/exp \# 3 'magnetization transfer imaging':ab,ti \# 4 mrcp: ab,ti OR mri: ab,ti OR nmr: ab,ti AND 
imaging: ab,ti OR fmri: ab,ti

\# 5 mrcp:ab,ti OR mri:ab,ti OR 'nmr imaging'ab, ti OR fmri:ab,ti

\# 6 cholangiopancreatograph*: ab,ti

\#7 \#1 OR \#2 OR \#3 OR \#4 OR \#5

\#8 \#6 OR \#7

\#9 'digestive system diseases'/exp

\#10 'biliary tract': ab,ti

\#11 'bile duct':ab,ti

\#12 'biliary duct':ab,ti

\#13 'jaundice':ab,ti

\#14 'stenosi':ab,ti OR 'strictur':ab,ti OR 'narrow': ab,ti OR 'explore':ab,ti OR 'exploration' $a b$,ti

\#15 'biliary stricture':ab,ti

\#16 \#10 OR \#11 OR \#12

\#17 \#14 AND \#16

\#18 \#13 OR \#15 OR \#17

\#19 \#9 OR \#18

\#20 \#8 AND \#19

\# 1 'nuclear magnetic resonance imaging': ab,ti \# 2 'nuclear magnetic resonance imaging'/exp \# 3 'magnetization transfer imaging':ab,ti \# 4 mrcp: ab,ti OR mri: ab,ti OR nmr: ab,ti AND imaging: ab,ti OR fmri: ab,ti

\# 5 mrcp:ab,ti OR mri:ab,ti OR 'nmr imaging'ab, ti OR fmri:ab,ti

\# 6 cholangiopancreatograph*: ab,ti

\#7 \#1 OR \#2 OR \#3 OR \#4 OR \#5

\#8 \#6 OR \#7

\#9 'digestive system diseases'/exp

\#10 'biliary tract': ab,ti

\#11 'bile duct':ab,ti

\#12 'biliary duct':ab,ti

\#13 'jaundice':ab,ti

\#14 'stenosi':ab,ti OR 'strictur':ab,ti OR 'narrow': ab,ti OR 'explore':ab,ti OR 'exploration':ab,ti

\#15 'biliary stricture':ab,ti

\#16 \#10 OR \#11 OR \#12

\#17 \#14 AND \#16

\#18 \#13 OR \#15 OR \#17

\#19 \#9 OR \#18

\#20 \#8 AND \#19

\#1 TS=((((Bile and (duct or tract) $)$ or biliary or pancreatic* or biliopancreatic*) and (tumor* or lesion* or injur* or strictur* or disease* or disorder* or obstruction* or stenos*)) or (Chronic pancreatitis or cholangitis or focal wall thickening or choledochal varices or (portal and (biliopathy or cavernoma)) 
or obstructive jaundice or bile leakage or retained stones or (bile duct and (intrahepatic or aberrant)) or intraductal masses or duct lumen))

\#2 TS=(((NMR or MR or protonspin or magnetization or MRI or chemical shift) and (imaging or tomography or scan)) or (magnetic resonance cholangiopancreatograph* or zeugmatograph* or functional MRI))

\#3 \#1 AND \#2

LILACS
1990 to the date of search.
\#1 (MH VALOR PREDITIVO DOS TESTES OR tw VALOR PREDITIVO DOS TESTES OR $\mathrm{MH}$ SENSIBILIDADE E ESPECIFICIDADE OR tw SENSIBILIDADE E ESPECIFICIDADE OR MH DIAGNOSTICO OR tw DIAGNOSTICO) OR ((tw sensitiv\$ OR tw sensibili\$ OR tw specific\$ OR tw especific\$) AND tw diagnos\$) OR ((tw predictiv\$ OR tw preditiv\$) AND (tw test\$ OR tw prueba\$))

\#2 mh ductos biliares OR mh bile ducts OR mh conductos biliares OR tw canais biliares OR tw ductos biliferos $\mathrm{OR}$ tw via biliar OR tw vias biliares OR ex a03.159.183\$ OR mh bile ducts, intrahepatic OR mh conductos biliares intrahepaticos OR tw canais biliares intra-hepaticos OR tw via biliar intra-hepatica OR tw vias biliares intra-hepaticas OR ex A03.620.150\$ OR mH bile ducts, extrahepatic $\mathrm{OR} \mathrm{mH}$ conductos biliares extrahepaticos $\mathrm{OR}$ $\mathrm{mH}$ ductos biliares extra-hepaticos OR tw sistema biliar extra-hepatico OR tw via biliar extra-hepatico OR tw vias biliares extra-hepaticos $\mathrm{OR} \mathrm{mH}$ bile ducts diseases OR $\mathrm{mH}$ enfermedades de los conductos biliares OR $\mathrm{mH}$ doenças dos ductos biliares OR tw doenças das vias biliares OR ex C06.130. 120\$ OR mH neoplasias dos ductos biliares OR $\mathrm{mH}$ bile duct neoplams OR $\mathrm{mH}$ neoplasias de los conductos biliares OR tw câncer dos ductos biliares OR tw câncer do ducto\$ biliar\$ OR tw neoplasias do ducto\$ biliar\$ OR tw tumores das vias OR ex C04.588.274.120.250\$ OR ex C06.130.120. $120 \$$ OR ex C06.130.320.120\$ OR C06.301.120. 250\$ OR mH choledocholithiasis OR mH coledocolitiasis OR $\mathrm{mH}$ coledocolitiase OR tw coletiase do ducto OR tw biliar comum OR tw colelitiase do colédoco OR ex C06.130.120.250.174\$C06.130. 409.267\$ OR mH gallstones OR mH cálculos biliares OR tw cálculos nas vias biliares OR tw calculo do trato biliar OR tw pedras no trato biliar 
OR tw cálculos na vesícula biliar OR tw pedras na vesícula biliar OR ex C06.130.409.633\$ OR ex C06.130.564.332.500\$ OR ex C23.300.175. 525\$ OR mH pancreatic neoplasms OR $\mathrm{mH}$ neoplasias pancreáticas OR tw câncer\$ do pâncrea \$ OR tw câncer pancreatic\$ OR tw tumor\$ pancrea\$ OR ex C04.588.274.761\$ OR ex C04.588.322. 421\$ OR ex C06.301.761\$ OR ex C06.689.667\$ OR C19.344.421\$

\#3 MH Cholangiopancreatography, magnetic resonance OR $\mathrm{mH}$ pancreatocolangiografia por resonancia magnética $\mathrm{OR} \mathrm{mH}$ colangiopancreatografia por ressonância magnética OR tw MRCP OR tw colangiopancreatograf\$ OR ex E01.370.350.500. $100 \$$ OR E01.370.350.825.500.100\$ OR E01. 370.372.207\$ OR mH cholangiography OR mH colangiografia OR mH colangiografia OR ex E01. 370.350.700.715.200\$ OR E01.370.372.200\$

\#4 MH magnetic resonance imaging OR mH imagen por resonancia magnética $\mathrm{OR} \mathrm{mH}$ imagem por ressonância magnética $\mathrm{OR}$ tw imagem por ressonância magnética OR tw imageamento por ressonância magnética OR tw imagem por chemical shift OR tw tomografia por RM OR tw tomografia por RMN OR tw tomografia por spin do próton OR tw varreduras por irm OR tw imagem contrastada por transferência de magnetização $\mathrm{OR}$ tw irmf OR tw image\$ por ressonanc\$ OR tw magnetic\$ funciona $\$$ OR tw irm funcional OR ex E01.370.350.500\$ OR E01.370.350.825\$ OR SP4.001.002.015.044.010.006\$ \#1 AND 2 AND 3 AND 4

Appendix 2. QUADAS-2 criteria definition for signalling questions

\begin{tabular}{|c|c|c|c|c|}
\hline Domain & Participant selection & Index test & Reference standard & Flow and timing \\
\hline $\begin{array}{l}\text { Signalling questions } \\
\text { and criteria }\end{array}$ & $\begin{array}{l}\text { 1. Was a consecutive or } \\
\text { random sample of par- } \\
\text { ticipants enrolled? } \\
\text { Answer: } \\
\text { Yes - if the study reported } \\
\text { on a consecutive or a ran- } \\
\text { dom selection of partici- } \\
\text { pants } \\
\text { No - if the study reported }\end{array}$ & $\begin{array}{l}\text { 1. Were the combi- } \\
\text { nation of MRCP and } \\
\text { MRI results interpreted } \\
\text { without knowledge of } \\
\text { the results of the refer- } \\
\text { ence standard? } \\
\text { Answer: } \\
\text { Yes - if the study re- } \\
\text { ported that the results }\end{array}$ & $\begin{array}{l}\text { 1. Was the pool of ref- } \\
\text { erence standards used } \\
\text { correctly in terms of the } \\
\text { target conditions, i.e., } \\
\text { ERCP, PTC, and en- } \\
\text { doscopic ultrasonogra- } \\
\text { phy for presence and } \\
\text { site of stenosis; surgery, } \\
\text { histopathology, }\end{array}$ & $\begin{array}{l}\text { 1. Was there an appro- } \\
\text { priate interval between } \\
\text { the index test and the } \\
\text { reference standard? } \\
\text { Answer: } \\
\text { Yes - if the interval be- } \\
\text { tween the index test and } \\
\text { the reference standard } \\
\text { was hours to } 7 \text { days, }\end{array}$ \\
\hline
\end{tabular}


on another form of selection of participants Unclear - if the study did not report on how the participants were enrolled

2. Did the study avoid inappropriate exclusions?

Answer:

Yes - if the study reported all exclusions and causes. No - if the study reported exclusions because of "non-diagnostic imaging by artefacts"

Unclear - if the study did not report causes of exclusions.

3. Were the participants with the target condition submitted to other imaging tests before the index test and reference standard?

Answer:

Yes - if other imaging tests were used (ultrasonography, computed tomography)

No - no other imaging tests were used.

Unclear - if the study does not report the use of other imaging tests of the combination of MRCP and MRI were interpreted without the knowledge of the results of the reference standard No - if the study reported that the results of the combination of MRCP and MRI were interpreted with the results of the reference standard

Unclear - if the study did not report information about blinding of the results of the combination of MRCP and MRI and reference standard

2. If a threshold was used, was it pre-speci-

\section{fied?}

Answer:

Yes - if the study reported a value to consider a bile duct dilation beyond stenosis No - if the study reported bile duct dilation without a pre-specified threshold

Unclear - if the study reported stenosis and the presence of bile duct dilatation, but the study was not clear if a threshold was used and clinical follow-up for the nature of stenosis (benign and malignant)?

Answer:

Yes - if studies used ERCP, PTC, and endoscopic ultrasonography for presence and site of stenosis, surgery, histopathology, and clinical follow-up for the nature of stenosis

No - if studies did not use the pool of reference standards for correct target conditions

Unclear - if studies did not specify how the pool of reference standards was used to define the target condition

2. Were the reference stan-

dard results interpreted without the knowledge of the results of the index test?

Answer:

Yes - if the study reported that the results of the reference standard were interpreted without the knowledge of the results of the index test No - if the study reported that the results of the reference standard were interpreted with the results of the test index Unclear - if the study did not report information about blinding of the results of the reference standard and the index test when the reference standard was not the clinical follow-up, and no less than 1 month when the reference standard was the clinical follow-up

No - if the interval was longer than 7 days when the reference standard was not the clinical follow-up, and less than 30 days, when the reference standard was the followup

Unclear - if the study did not report the interval between the index test and the reference standard

2. Did all study participants receive a reference standard?

Answer:

Yes - if all participants received a reference standard.

No - if there were withdrawals because some participants did not receive the reference standard

Unclear - if the study did not report if all participants received the reference standard or not

3. Did all participants receive the same reference standard?

Answer:

Yes - if the study had only 1 reference standard (ERCP).

No - if the study had more than 1 reference standard.

Unclear - if the study was 
not clear about the reference standard used

4. Were all participants included in the analysis?

Answer:

Yes - if all participants were included in the analysis.

No - if more than 1 participant was excluded from the analysis no matter the reason

Unclear - if it is not clear about the exclusions of participants from the analysis

\section{Risk of bias}

\section{Could the selection of participants have in- troduced bias?}

Answer:

High - if participants were enrolled retrospectively, if the study did not report the causes of exclusions, and if other imaging tests were used before the index test or reference standard

Low - if the study was in a consecutive or a random selection of participants, if participants were enrolled prospectively, and if the study described exclusions

Unclear - the study was not clear about the design, if the exclusions were not reported, and there was no information about if participants were submitted or not to other imaging tests
1. Could the conduct or interpretation of the combination of MRCP and MRI have introduced bias?

Answer:

High - the study reported that results of the combination of MRCP and MRI were interpreted with the results of reference standard Low - the study reported that results of combination of MRCP and MRI were interpreted without knowledge of the results of reference standard and there was a threshold to consider a bile duct dilation

Unclear - the study reported no information about blinding of the results of the reference standard and the combination of MRCP and MRI
1. Could the reference standard, its conduct, or its interpretation have introduced bias?

Answer:

High - the results of reference standard were interpreted with the results of the index test, and when the study used a reference standard that did not classify the target condition correctly, and the pool of reference standards was not used for the correct target condition Low - the results of the reference standard were interpreted without knowledge of the results of the index test, and ERCP alone or in combination with other tests classified and used the target condition correctly

Unclear - the study was not clear about the
1. Could the participant flow have introduced bias?

Answer:

High - the study used more than 1 reference standard and the time between the index test and reference standard was not clear

Low - the study used only 1 reference standard and all participants were included in the analysis Unclear - the study did not report if all participants received the reference standard, if the study did not report the interval between the reference standard and the index test 
blinding with the results of the reference standard and the index test Concerns about appli-
cability
1. Are there concerns that the included participants do not match the review question?

Answer:

High - if participants were enrolled for an investigation other than for suspicion of bile duct stenosis

Low - if participants were enrolled for investigation of suspicion of bile duct stenosis

Unclear - if the study did not report why the participants were enrolled in the study
1. Are there concerns that the combination of MRCP and MRI, its conduct, or its interpretation differ from the review question? Answer:

High - if the study did not report how the combination of MRCP and MRI was technically conducted and which magnetic field strength was used

Low - if the combination of MRCP and MRI results classified the target condition appropriately, if the study reported how the

combination of MRCP and MRI was technically conducted and if field strengths of $1.5 \mathrm{~T}$ or 3.0 $\mathrm{T}$ were used Unclear - insufficient data about the combination of MRCP and MRI were reported
1. Are there concerns that the target condition as defined by the reference standard does not match the review question?

Answer:

High - if there was no information how the reference standard or a pool of reference standards defined the bile duct stenosis and bile duct dilatation

Low - if the study reported how the reference standard or a pool of reference standards defined the bile duct stenosis and bile duct dilation

Unclear - the study did not report sufficient data on how the reference standard or a pool of reference standards defined the target condition

Footnotes:

- The reference standard for detection of presence and site of stenosis could only be ERCP or a combination of reference standards; however, ERCP should always be part of this combination.

- The reference standard to differentiate benign from malignant stenosis will be a combination of tests as clinical follow-up, surgical exploration, and histopathology.

- Studies with more than one objective may have more than one reference standard.

ERCP: endoscopic retrograde cholangiopancreatography; MRCP: magnetic resonance cholangiopancreatography; MRI: magnetic resonance imaging; PTC: percutaneous transhepatic cholangiography 


\section{WHAT'S NEW}

Last assessed as up-to-date: 7 March 2013.

Date Event Description

15 May 2015 Amended Contact details updated.

\section{CONTRIBUTIONS OFAUTHORS}

Clinical content expert: Giuseppe D’Ippolito and Augusto José Cavalcanti Neto.

Systematic review authors: Delcio Matos, Suzana Angélica Silva Lustosa, Augusto José Cavalcanti Neto, Caroline Nunes Reis, and Marcelle Procopio.

Methodologist: Andreia Cristina Feitosa do Carmo.

Statistician: Giovanni Casazza.

All review authors approved the content of the protocol.

\section{DECLARATIONSOF INTEREST}

None known.

\section{SOURCES OF SUPPORT}

\section{Internal sources}

- CAPES (Coordenação de Aperfeiçoamento de Pessoal de Nível Superior), Brazil.

Post-graduation Scholarship

\section{External sources}

- No sources of support supplied 